\title{
Ultrasound images and postnatal clinical outcomes in primary and secondary abnormalities of fetal brain (series of 53 cases)
}

\begin{abstract}
The objective of study is the analysis of ultrasound (US) images, clinical perinatal and long-term results in different types of primary and secondary abnormalities of the fetal brain. The article contains ultrasound (US) images and postnatal results in 53 fetuses with brain abnormalities detected after ultrasound anomaly scan. The features of fetuses with brain abnormalities were studied and archived. After receiving data about the postnatal catamnesis, a retrospective detailing of antenatal images was conducted. Adverse general postnatal outcome (GPO) meant perinatal or infant death; adverse clinical postnatal outcome (CPO) meant persistent neurological morbidity. In the studied series various options of US fetal brain anomalies were accompanied by a high frequency of adverse GPO (28/53 or $52.8 \%$ ), and adverse CPO with severe persistent neurologic morbidity (13/53 or $24.5 \%)$. US images and postnatal results in prosencephalic malformations, abnormalities of posterior fossa, destructive brain lesions, callosal agenesis and cortical dysplasia have been present as well as a number of distress-associated and infection-associated brain features as intraventricular hemorrhage, periventricular leukomalacia and cortical necrosis. A number of fetal intracranial changes were observed both in fetuses with infection and severe placental dysfunction. Most of such anomalies had periventricular or subependymal localization, which could associated with universal pathogenetic mechanism of the germinal matrix injury. Secondary changes of fetal brain occurred three times more often than primary, which confirms the opinion about the need of the third trimester pregnancy routine scan. This data would help to improve the quality of prenatal counseling and to select newborns for follow-up monitoring.
\end{abstract}

Keywords: fetus, brain, ultrasound, postnatal outcome
Volume 6 Issue I - 2017

\author{
Inessa Safonova,' Irina Lukjanova, ${ }^{2}$ Olena \\ Zhadan ${ }^{2}$ \\ 'Kharkiv Medical Academy of Postgraduate Education, Ukraine \\ ${ }^{2}$ Institute of Pediatrics, Obstetrics and Gynaecology of Academy \\ of Medical Science of Ukraine, Ukraine
}

Correspondence: Inessa Safonova, Kharkiv Medical Academy of Postgraduate Education, Ukraine, Tel +380505225989, Email inessa7799@gmail.com

Received: December 22, 2016 | Published: January 03, 2017

\section{Introduction}

Nowadays, in publications which dedicated to ultrasound in obstetrics a format that represents series of cases with their ultrasound images and clinical features and results is actual. ${ }^{1,2}$ It is caused by difficulties of adequate prognosis of postnatal result, though exactly this prediction is the main task of prenatal consultation given to a pregnant woman with suspicion of abnormal development of the fetus central nervous system (CNS). Such anomalies of fetal CNS as agenesis of the corpus callosum and cortical displasia, found mainly in the second half of pregnancy, after 20-26weeks. ${ }^{3}$ The perinatal prognosis in some forms of antenatal fetal brain lesions is definitely unfavorable. But the information about the child development prognosis with moderate development of prosencephalon disorder, as well as in isolated and associated callosal anomalies, some kinds of the posterior fossa anomalies, hydrocephalic syndrome is contradictory and indefinite. ${ }^{2}$ Also, there are difficult issues for prediction results in focal tumor and cystic antenatal brain lesions. ${ }^{3}$ Diagnostics of brain lesions at the antenatal stage with a target NSG research allows to find fetuses with the necessity of postnatal observation. In the last decade, a considerable number of publications are dedicated to diagnosis of secondary fetal brain damages with infection processes, or placenta disorders. ${ }^{4,5}$ Periventricular leukomalacia (PVL) is diffuse change of the periventricular myelin density. It's diagnosis takes a special place in perinatal neurosonography (NSG). Antenatal PVL reports are rarely published. The research results have showed that damage of the white matter of brain is the most important prenatally identifiable risk factor for cerebral paralysis development. ${ }^{6-9}$ The objective of study is the analysis of ultrasound (US) images, clinical perinatal and longterm results in different types of primary and secondary abnormalities of the fetal brain.

\section{Case presentation}

Prospective single-center observational study was performed. The features of 53 fetuses with brain abnormalities which were detected after 22gestational weeks were studied and archived. After receiving data about the postnatal catamnesis, a retrospective detailing of antenatal images was conducted. US exams and archiving of US images were made on devices Voluson 730pro (GE, USA) and Philips HD 11 (Germany). In all cases, etiopathogenic and clinical perinatal features were studied. Antenatal diagnosis was verified by magnetic resonance tomography (MRI) and computerized tomography scan (CT scan) in 11 infants and by NSG in 20 infants and by autopsy in 28 cases. Postnatal results were classified in the next way: adverse general postnatal outcome (GPO) perinatal or infant death, adverse clinical postnatal outcome (CPO) serious postnatal morbidity as persistent neurological disorders. In cases of perinatal mortality pathologic diagnosis was studied. Long-term consequences of pathological changes identified on the antenatal stage were elucidated by a telephone survey of mothers in 12 months after childbirth. In series were not included cases of anencephaly-acrania and exencephaly like anomalies that have definitely adverse perinatal result and cysts of chorionic plexus which identified in middle trimester and subsequently regressed. 
The study was approved by the Kharkiv Regional Hospital with Regional Perinatal Center Research Subjects Review Ethical Board. Figure 1-12 present fetal and newborns images and data about postnatal clinical catamnesis.

All cases with prosencephalic malformations had an adverse catamnesis connected with combined pathology. Two cases of alobar holoprosencephaly (HPE) and syndromic semilobar HPE were connected with perinatal losses. Syndromic lobar HPE with partial callosal agenesis and septo-optic dysplasia didn't have perinatal consequences, but the clinical outcome for the child was unfavorable as well (Figure1).

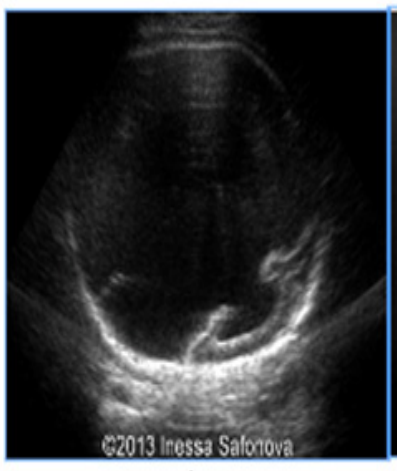

A
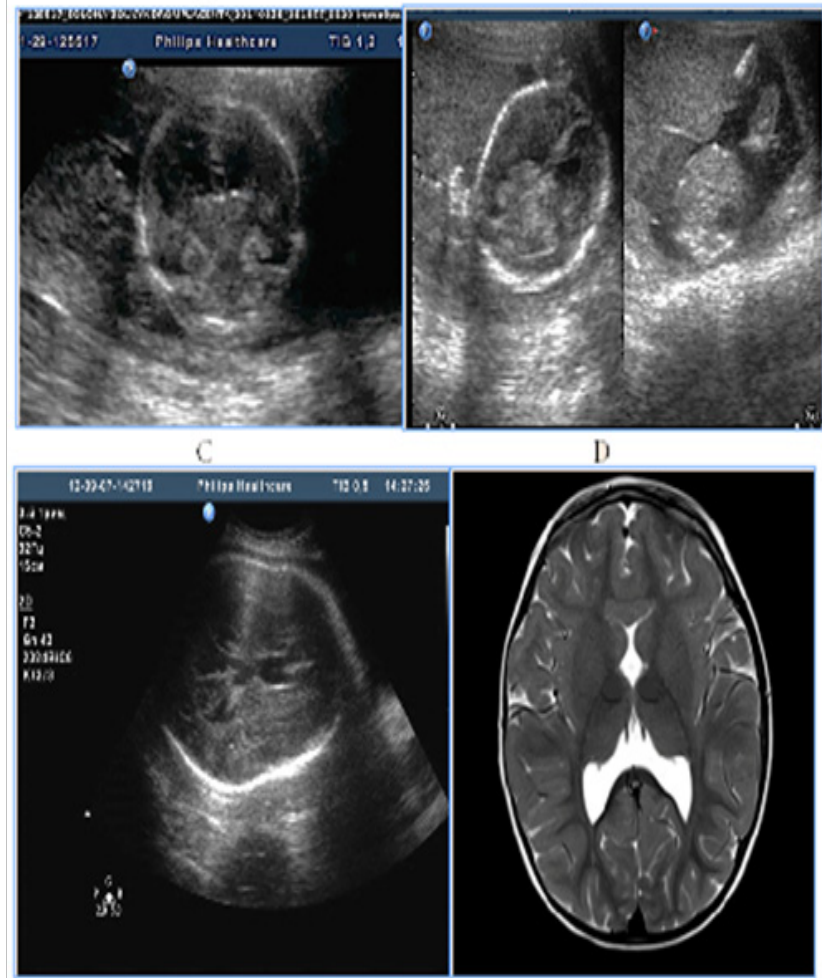

E.
D

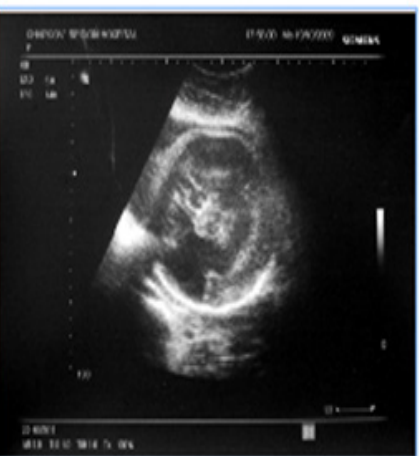

B

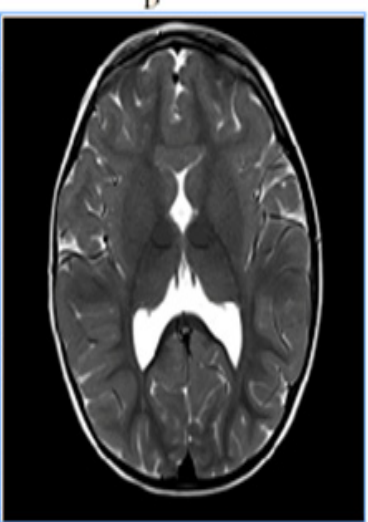

Figure I HPE in 3rd trimester, 4cases.

HPE in $3^{\text {rd }}$ trimester, 4 cases:

A) Alobar syndromic HPE with the residual mantle of the cortex at the base of the brain, severe progressive disgenetic hydrocephalus with macrocrania, intranatal death, clinical intranatal mismatch of head and pelvic size, fetus-damaging operation.
B) Isolated alobar HPE, cup-shaped type, the biggest part of the cortical mantleis attended, but it does not cover a single anterior ventricular area, infant death.

C,D,E). Semilobar syndromic HPE with callosal agenesis. The ventricles are connecting through a narrow channel across the midline, thalamuses partially fused, IUGR, absent umbilical enddiastolic flow, antenatal death.

F). Fetal NSG and MRI of the newborn with syndromic lobar HPE, partial callosal agenesis, septo-optic dysplasia, US variant with merged, widely separated narrow acute-angled anterior horns of ventricular and dilated Verga's cavity, long-term neurological disorders, severe visual impairment, epilepsy in the kid.

In the 3 cases of mild and moderate fetal ventriculomegaly the adverse GPO was observed in 1 case, adverse CPO- in the other two,so any expression of the fetal hydrocephalic syndrome requires postnatal catamnesis observation (Figure 2)

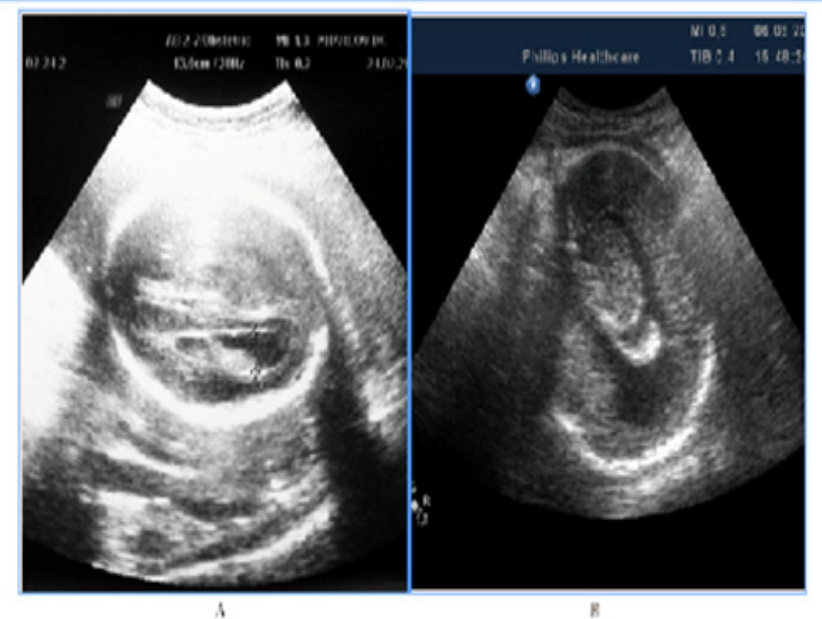

Figure 2 Mild and moderate fetal ventriculomegaly, 3cases.

Mild and moderate fetal ventriculomegaly, 3cases. A). Mild (border) fetal ventriculomegaly, the lateral ventricles thresholds expanding up to $11 \mathrm{~mm}$, without any clinical manifestations in neonatal stage, long-term neurological disorders by the end of the $2^{\text {nd }}$ year age. B). Lateral ventricles are $15 \mathrm{~mm}$ width in fetus with associated moderate hydrocephalus, infant death.

In 3 cases of lumbosacral spinal dysraphism we observed one adverse GPO in moderate obstructive hydrocephalus and also there were two cases with adverse $\mathrm{CPO}$ at severe hydrocephalus.

Obviously, that GPO of hydranencephaly and schizencephaly were unfavorable, wherein only bilateral schizencephaly was accompanied with clinically significant macrocrania (head circumference $430 \mathrm{~mm}$, it was above $95^{\text {th }}$ percents of the average gestational circumference) (Figure 3). In case of hydranencephaly the liquor pressure did not increase and remained normal size of the fetal skull.

Antenatal US signs of posterior fossa anomalies in 3 of 4 cases had favorable GPO, 2 of 4 had favorable CPO. That's why, for such anomalies, antenatal prognosis could contradict with clinical postnatal catamnesis, and cause difficulties with accurately prognosing. In this way, abnormalities of Dandy-Walker spectrum are associated with indefinite perinatal and postnatal risk (Figure 4). 


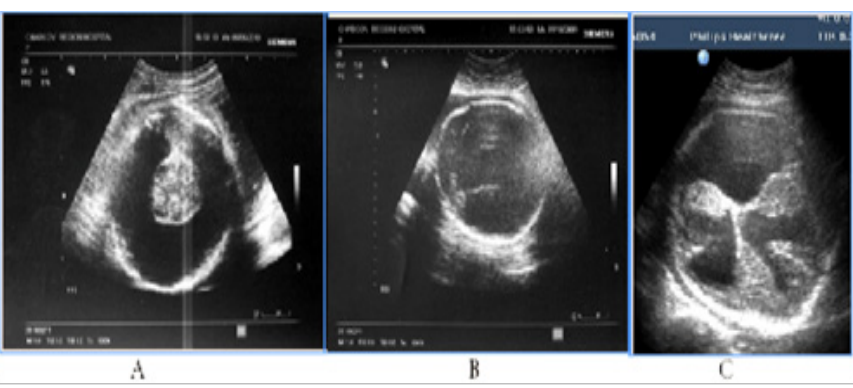

Figure 3 Destructive brain lesions, 3 cases.

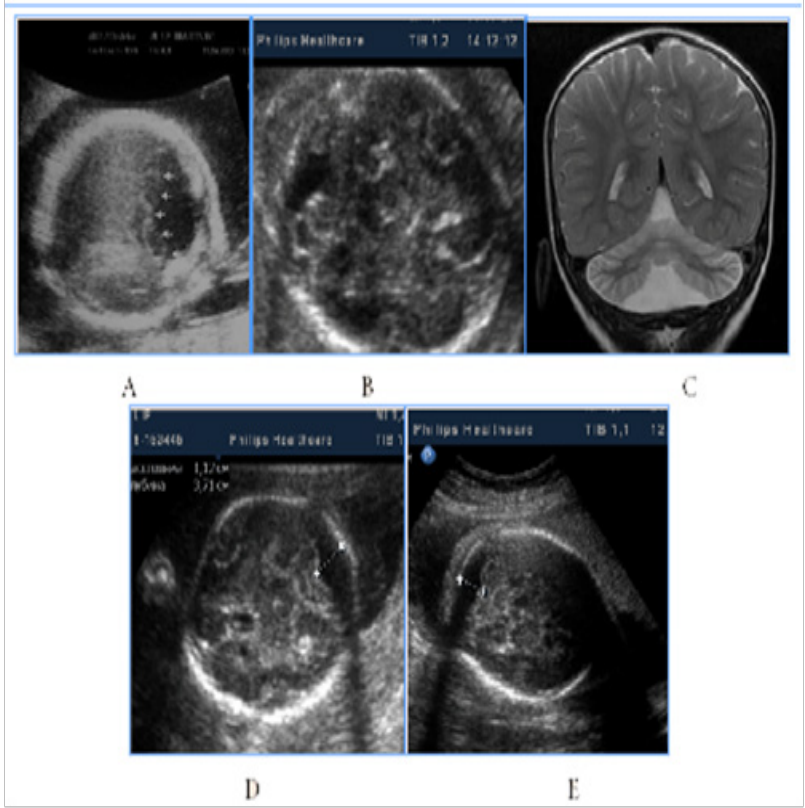

Figure 4 US images of the fetuses posterior fossa structures and postnatal results, 4 cases.

Figure 3 Destructive brain lesions, 3 cases. A) Hydranencephaly, total absence of cortical mantle and falx, saved diencephalon, hypothalamus and basal ganglia, wherein postnatal life was possible, by maintaining thermoregulatory functions, catamnesis - infant death in 3months. B) No structures of the brainstem, there are only remnants of supratentorial tissue, no sickle and cortical border, intranatal fetal death. C) Bilateral schizencephaly (type II, "open lip") with severe hydrocephalus and macrocrania, intranatal fetal death, clinically narrow pelvis, fetus-damaging operation.

Figure 4 US images of the fetuses posterior fossa structures and postnatal results, 4cases. A) Malformation or Dandy-Walker complex, agenesis of the cerebellar vermis and cystic expansion of the fourth ventricle in conjunction with facial and cardiac abnormalities, late neonatal death. B, C) Fetal US and brain MRI after birth in isolated Dandy-Walker variant, worms hypoplasia and mega-cisterna magna, favorable GPO and CPO. D) Mega-cisterna magna associated with facial and cardiac abnormalities, neonatal complications, long-term neurological disorders. E) Isolated mega-cisterna magna, favorable postnatal and long-term outcome.

Postnatal result of fetal brain with cysts and tumors depend on the destruction degree of the brain tissue, as well as the severity of the mass effect. Two our observations have shown that even with extensive changes there are possible favorable GPO and CPO, if the destructive lesions are absent. Antenatal detection facilitates timely initiation of treatment with erased or absent clinical symptoms (Figure $5)$.
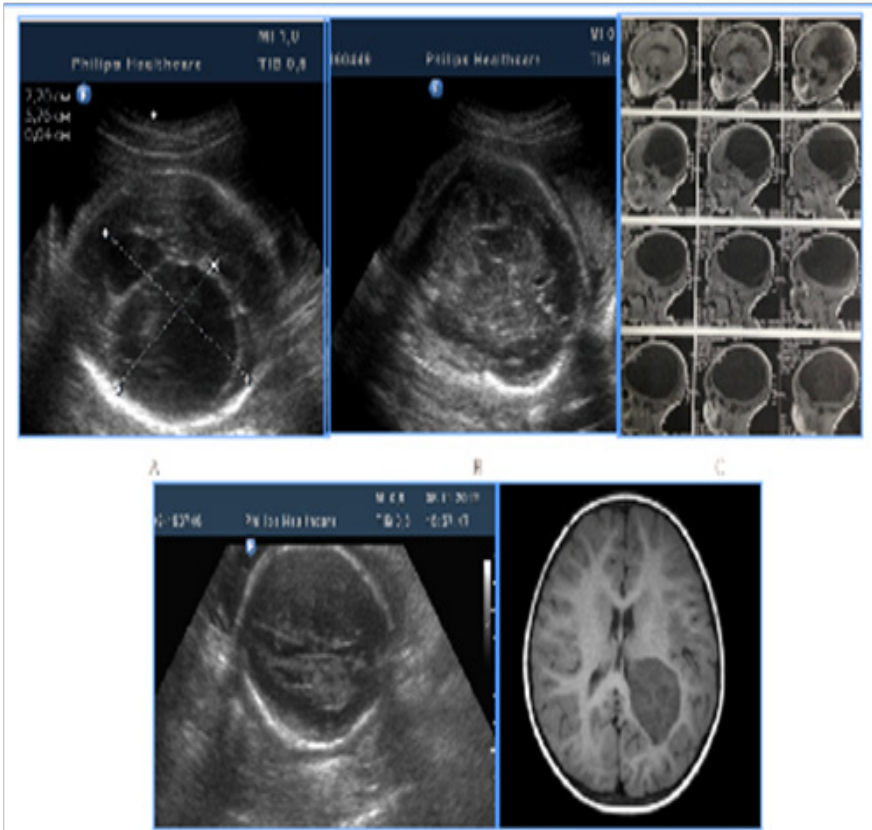

Figure 5 Brain cyst and brain mass and favorable postnatal results, fetal US and brain MRI after birth, 2cases.

Brain cyst and brain mass and favorable postnatal results, fetal US and brain MRI after birth, 2 cases: A,B,C) Giant arachnoid cyst of the brain hemisphere origing from Sylvian fissure, macrocrania. No signs of compression and dysplasia of thalamus, midbrain, brainstem, and cerebellum, elective surgical delivery, shunting surgery was performed in the neonatal period due to clinically significant compression; favorable GPO and CPO. D) Choroid plexus papilloma, surgery after birth - favorable GPO and CPO.

7 cases of callosal agenesis appeared with various echographic variations and associations (Figure 1C) (Figure 1F) (Figure 6) with colpocephaly, interhemispheric cyst, hydrocephalus, absence of the cavum septi pellucidi, as well as with semilobar and lobar HPE and septo-optic dysplasia. From the 7cases of antenatal detection of callosal agenesis, poor GPO were observed in 2cases, poor CPO in 3 cases, so postnatal outcome directly depends on the availability of the associated anomalies.

Figure 6 Callosal agenesis, different sonographic variants and catamnesis. A) Associated callosal agenesis combined with colpocephaly ("tear-drop" configuration of the posterior horns of the lateral ventricles), progressive moderate hydrocephalus, without neonatal complications, long-term severe neurological disorders and neuro-psychological retardation. B) Moderate hydrocephalus, partial agenesis of the corpus callosum and subependymal cyst, infant death in 5months. C) Syndromal callosal agenesis, sonographic variant with absence of the cavum septi pellucidi and ventriculomegaly, neonatal death. D) Isolated callosal agenesis with an expanded and elevated third ventricle in the form of the "inter-hemispheric cyst», no neonatal and infant complications.

In 5 fetuses with severe prolonged course of placental dysfunction we antenatally detected modified insular complex with anomalously broad lateral (Sylvian) sulcus as a signs of abnormal gyration and 
sulcation (Figure 7). US images of cortical dysplasia in our study were associated with fetal intrauterine growth retardation (IGR), Oligohydramnios, metabolic acidosis and neuro infection. These fetuses had adverse GPO (3cases) and extremely unfavorable CPO (2cases) (Figure 8).

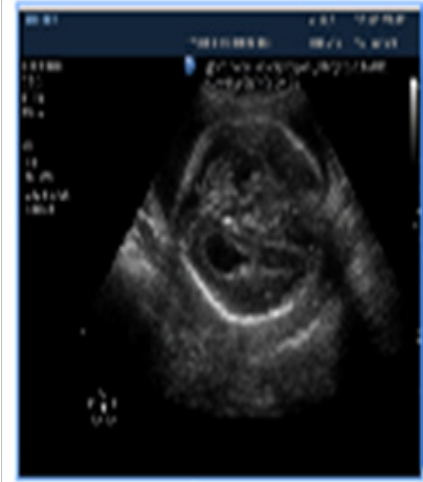

$A$

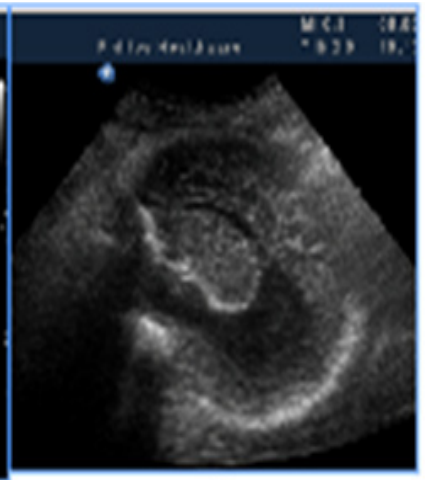

B

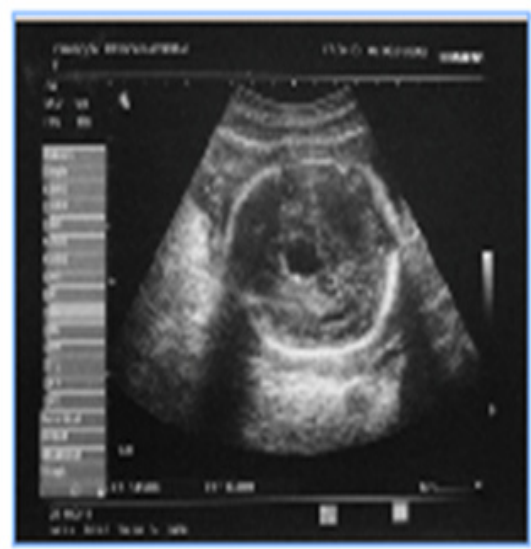

CD

Figure 6 Callosal agenesis, different sonographic variants and catamnesis.

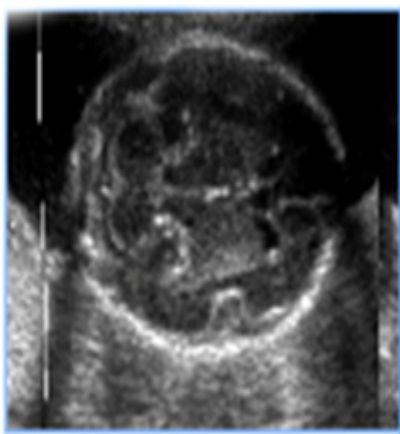

$A$

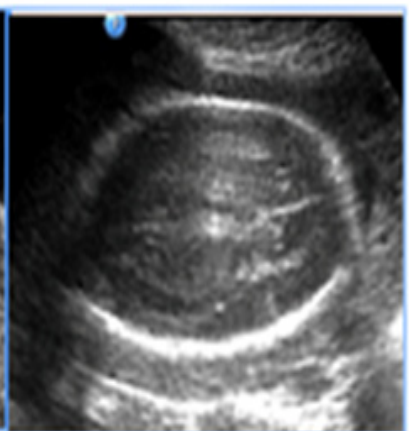

B
Figure 7 NSG of two fetuses of the same gestational age (29 GW) with normal brain image $(A)$, also with a signs of cortical dysplasia (B).

NSG of two fetuses of the same gestational age (29 GW) with normal brain image (A), also with a signs of cortical dysplasia (B).

Figure 8 Abnormal image of the cerebral cortex as a manifestation of abnormal neuronal migration in two fetuses in 3rd trimester of pregnancy, fetal US and neonatal MRI. A, B) 30GW, cortical dysplasia, the type of neuronal heterotopia and pachygyria, long-term violations as severe epilepsy and retardation of development in catamnesis. C,D,E) $29 \mathrm{GW}$, cortical dysplasia in the fetus with significant IGR and antenatal distress, postnatal transmantian dysplasia, lissencephaly and presilvian polymicrogyria that clinically was accompanied by hypotension and child neurological disabilities.

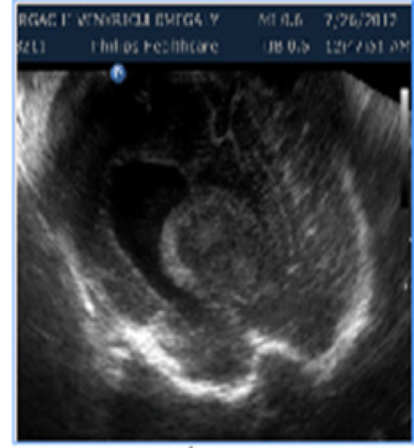

$A$

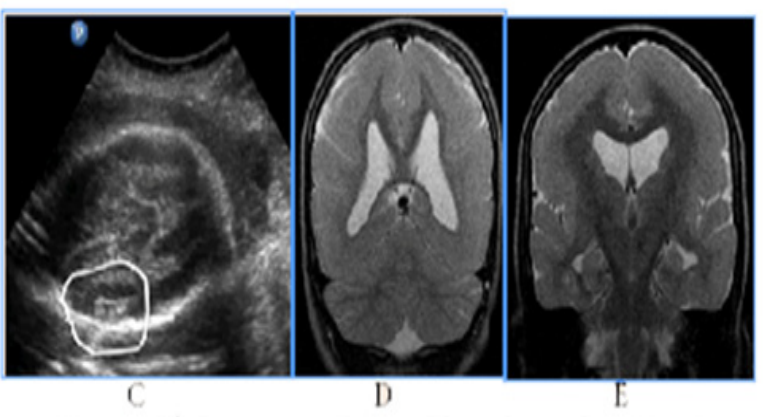

Figure 8 Abnormal image of the cerebral cortex as a manifestation of abnormal neuronal migration in two fetuses in $3 r$ trimester of pregnancy, fetal US and neonatal MRI.

To distress associated antenatal US features of fetal brain could include signs of cortical dysplasia, antenatal intraventricular hemorrhage and PVL (Figure 9) (Figure 10). PVL in the form of small cyst was observed in two fetuses with different premorbid background one fetus with severe prenatal distress and the others with neuroinfection. Particularly attention deserved severe postnatal aftermath of fuzziness and reduced contrast of the fetal brain structures due to cerebral edema and cortical laminar necrosis at 5 cases which had different pathogenetic background (Figure 10). Such contrast of structures looked like a result of wrong exposure while getting a picture, but thats were real images, with progression of edema structures looked absolutely "blurred" and cystic PVL lesions were detected. Postnatal catamnesis of all cases was extremely unfavorable.

Among of infection-associated echographic signs of the fetal brain anomalies (for CMV, maternal VIH/AIDS, also unclassified etiology of neuroinfection) there were revealed changes, mainly of the intraventricular, subependymal and periventricular localization (Figure 11) (Figure 12) subependymal cysts, heterogeneous periventricular echogenicity, intraventricular adhesions, antenatal intraventricular haemorrage, hydrocephalus, cerebral edema signs, cystic PVL and signs of disgenetic processes - partial agenesis of the corpus callosum and cortical dysplasia.

A number of fetal intracranial changes as cortical dysplasia, intraventricular hemorrhage, PVL and reduced contrast of the 
fetal brain structures were observed both in infection and in severe placental dysfunction. Most of such anomalies had periventricular or subependymal localization, which could associated with universal polyetiologic pathogenetic mechanism of the germinal matrix injury. Figure 9 Antenatal distress associated sonographic brain features. A) Revers diastolic flow in the umbilical artery. B). fuzziness and reduced contrast of the fetal brain structures due to cerebral edema on the background of antenatal distress and metabolic acidosis. In clinical catamnesis perinatal loss and extremely severe persistent neurologic morbidity.

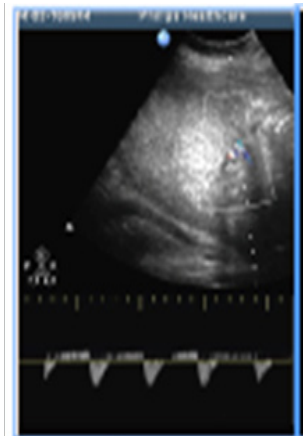

$A$
B

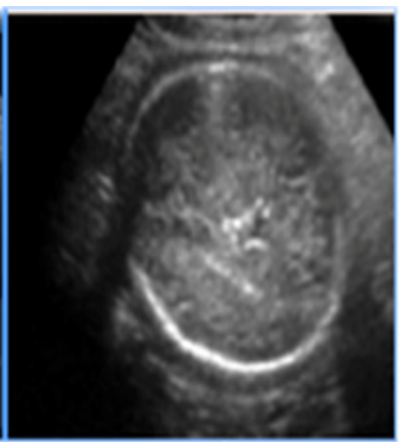

Figure 9 Antenatal distress associated sonographic brain features.
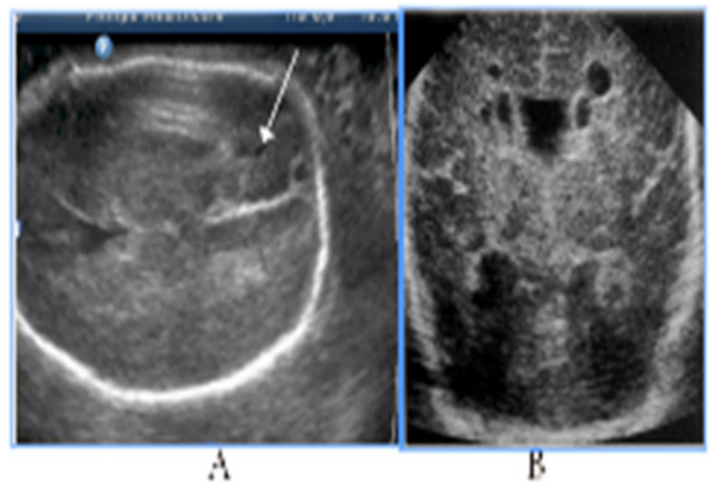

Figure I0 Fetal distress associated small-cystic PVL in the fetus and newborn.

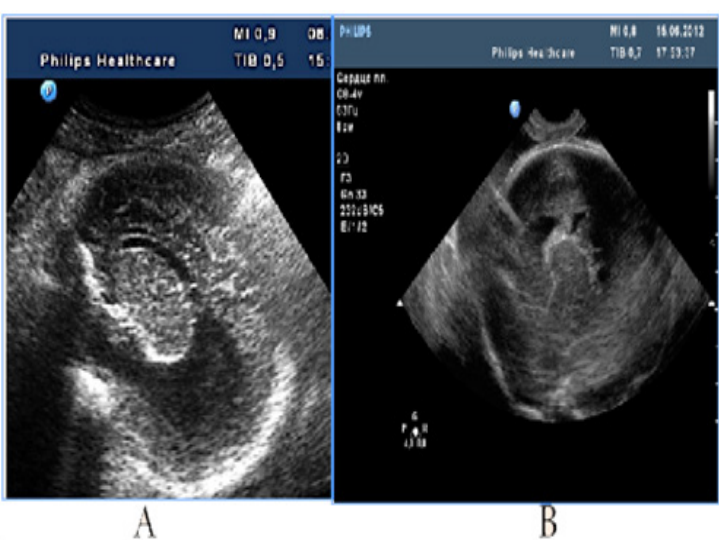

Figure II Infection-associated fetal brain abnormalities (neuroinfection of unknown etiology), two cases.

Figure 10 Fetal distress associated small-cystic PVL in the fetus and newborn. A) $31 \mathrm{GW}$, severe IGR, fetal metabolic acidosis, fuzziness and reduced contrast of the brain structures due to cerebral edema, skull deformation due to cortical necrosis, small cysts PVL in the intermediate zone (arrow). B) NSG of the newborn's brain of on the 2nd day of life, progressive small-cystic PVL. Neonatal death.

Figure 11 Infection-associated fetal brain abnormalities (neuroinfection of unknown etiology), two cases: A) Hydrocephalus, partial agenesis of the corpus callosum and subependymal cyst, infant death in 5 months. B) Intraventricular adhesion, adverse CPO.

Figure 12 Infection-associated fetal brain abnormalities (maternal VIH/AIDS), fetal NSG and postnatal MRI scans. A) Significant fetal hydrocephalus with a total extension of the ventricular system, cystic PVL (arrow). B) MRI after birth, cystic lesions on both sides of the shell, progressive internal and external hydrocephalia. Neonatal death.

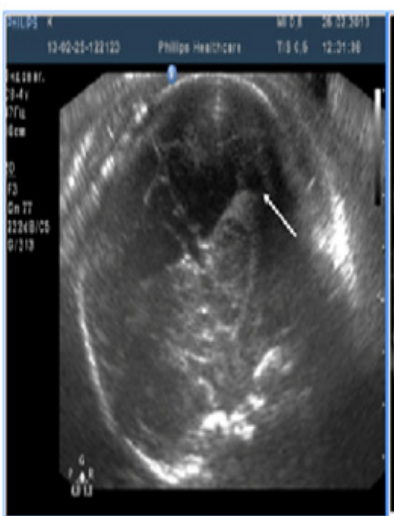

A

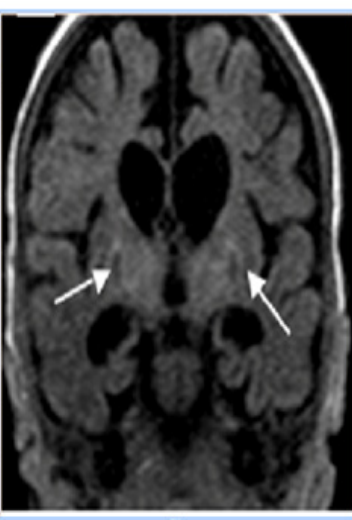

B
Figure 12 Infection-associated fetal brain abnormalities (maternalVIH/AIDS), fetal NSG and postnatal MRI scans.

\section{Discussion}

In the studied series of 53 cases various options of US fetal brain anomalies, which were detected during the second half of pregnancy, were accompanied by a high frequency of adverse GPO (28/53 or $52.8 \%$ ), and adverse CPO with severe persistent neurologic morbidity $(13 / 53$ or $24.5 \%)$. Perinatal results, both general and clinical, were worse in associated anomalies than in isolated ones.

Secondary changes of fetal brain occurred three times more often than primary, which confirms the opinion about the need of the third trimester pregnancy routine scan.

A number of fetal intracranial changes as cortical dysplasia, intraventricular hemorrhage, PVL and reduced contrast of the fetal brain structures were observed both in fetuses with infection and severe placental dysfunction. Most of such anomalies had periventricular or subependymal localization, which could associated with universal pathogenetic mechanism of the germinal matrix injury.

It seems necessary to set up multi-center studies, and development of specific web resources, which would allow to archive and analyze the abnormal sonographic images, and helps to identify and demonstrate their relationship with postnatal neurological disease. All this would help to improve the quality of prenatal counseling and to select newborns for follow-up monitoring.

\section{Acknowledgements}

The abstract including data in this article were presented at the 
$24^{\text {th }}$ World Congress on Ultrasound in Obstetrics and gynecology, 1417Sept, Barselona, Spain.

\section{Conflict of interest}

The authors declare that there is no conflict of interests regarding the publication of this paper.

\section{References}

1. Safonova I, Lukjanova I, Zhadan E, et al. Fetal brain anomalies associated with intrauterine neuroinfection and fetal distress and their postnata results. Radiation Diagnostics Radiation Therapy. 2014;(3-4):44-54.

2. De Catte L, De Keersmaeker B, Claus F. Prenatal neurologic anomalies:sonographic diagnosis and treatment. Paediatr Drugs. 2012;14(3):143155.

3. Isaacs H. Fetal brain tumors:a review of 154 cases. Am J Perinatol. 2009;26(6):453-466.

4. Reuter KL, Mc Gahan JP. Obstetric and Gynecologic Ultrasound. Case Review Series: Elsevier Health Sciences; 2013. 272 p.
5. Benoist G, Salomon LJ, Mohlo M, et al. Cytomegalovirus-related fetal brain lesions: comparison between targeted ultrasound examination and magnetic resonance imaging. Ultrasound Obstet Gynecol. 2008;32(7):900-905.

6. Pugash D, Mayer C, Hendson G, et al. Periventricular leukomalacia in the fetus: prenatal imaging and diagnostic features. Ultrasound in $\mathrm{Ob}$ stetrics \& Gynecology. World Congress on Ultrasound in Obstetrics and Gynecology. 2013;42(Suppl. 1):1-47.

7. Safonova I. Fetal brain anomalies assotiated with intrauterine neuroinfection and fetal distress and their postnatal results. Ultrasound Obstetrics and Gynecology V. 2014;44(S1):101.

8. Schell-Apacik CC, Wagner K, Bihler M, et al. Agenesis and dysgenesis of the corpus callosum: clinical, genetics and neuroimaging findings in a series of 41 patients. Am J Med Genet. 2008;146(19):2501-2511.

9. Smith T, Tekes A, Boltshauser B, et al. Comissural malformations: beyond the corpus callosum. J Neuroradiol. 2008;35(5):301-303. 